\title{
Repair of Acute Respiratory Distress Syndrome by Stromal Cell Administration in COVID-19 (REALIST-COVID-19): A structured summary of a study protocol for a randomised, controlled trial
}

\author{
Ellen Gorman ${ }^{1}\left(\mathbb{D}\right.$, Manu Shankar-Hari ${ }^{2}$, Phil Hopkins ${ }^{3}$, William S. Tunnicliffe ${ }^{4}$, Gavin D. Perkins ${ }^{5}$, \\ Jonathan Silversides ${ }^{6}$, Peter McGuigan ${ }^{7}$, Colette Jackson ${ }^{8}$, Roisin Boyle $^{8}$, Jamie McFerran $^{8}$, Cliona McDowell $^{8}$, \\ Christina Campbell ${ }^{8}$, Margaret McFarland ${ }^{6}$, Jon Smythe ${ }^{9}$, Jacqui Thompson ${ }^{9}$, Barry Williams ${ }^{10}$, Gerard Curley ${ }^{11}$, \\ John G. Laffey ${ }^{12}$, Mike Clarke ${ }^{13}$, Cecilia O'Kane ${ }^{1}$ and Daniel F. McAuley ${ }^{1 *}$
}

\begin{abstract}
Objectives: The primary objective of the study is to assess the safety of a single intravenous infusion of Mesenchymal Stromal Cells (MSCs) in patients with Acute Respiratory Distress Syndrome (ARDS) due to COVID-19. Secondary objectives are to determine the effects of MSCs on important clinical outcomes, as described below.

Trial design: REALIST COVID 19 is a randomised, placebo-controlled, triple blinded trial.

Participants: The study will be conducted in Intensive Care Units in hospitals across the United Kingdom. Patients with moderate to severe ARDS as defined by the Berlin definition, receiving invasive mechanical ventilation and with a diagnosis of COVID-19 based on clinical diagnosis or PCR test will be eligible.

Patients will be excluded for the following reasons: more than 72 hours from the onset of ARDS; age < 16 years; patient known to be pregnant; major trauma in previous 5 days; presence of any active malignancy (other than non-melanoma skin cancer); WHO Class III or IV pulmonary hypertension; venous thromboembolism currently receiving anti-coagulation or within the past 3 months; patient receiving extracorporeal life support; severe chronic liver disease (Child-Pugh > 12); Do Not Attempt Resuscitation order in place; treatment withdrawal imminent within 24 hours; prisoners; declined consent; non-English speaking patients or those who do not adequately understand verbal or written information unless an interpreter is available; previously enrolled in the REALIST trial.

* Correspondence: d.f.mcauley@qub.ac.uk

'Wellcome Wolfson Institute for Experimental Medicine, School of Medicine, Dentistry and Biomedical Science Queen's University Belfast, Belfast, UK

Full list of author information is available at the end of the article

(c) The Author(s). 2020 Open Access This article is licensed under a Creative Commons Attribution 4.0 International License, which permits use, sharing, adaptation, distribution and reproduction in any medium or format, as long as you give appropriate credit to the original author(s) and the source, provide a link to the Creative Commons licence, and indicate if changes were made. The images or other third party material in this article are included in the article's Creative Commons licence, unless indicated otherwise in a credit line to the material. If material is not included in the article's Creative Commons licence and your intended use is not permitted by statutory regulation or exceeds the permitted use, you will need to obtain permission directly from the copyright holder. To view a copy of this licence, visit http://creativecommons.org/licenses/by/4.0/. The Creative Commons Public Domain Dedication waiver (http://creativecommons.org/publicdomain/zero/1.0/) applies to the data made available in this article, unless otherwise stated in a credit line to the data. 
(Continued from previous page)

Intervention and comparator: Intervention: Allogeneic donor CD362 enriched human umbilical cord derived mesenchymal stromal cells (REALIST ORBCEL-C) supplied as sterile, single-use cryopreserved cell suspension of a fixed dose of $400 \times 10^{6}$ cells in $40 \mathrm{ml}$ volume, to be diluted in Plasma-Lyte 148 to a total volume of $200 \mathrm{mls}$ for administration.

Comparator (placebo): Plasma-Lyte 148 Solution for Infusion (200m/s).

The cellular product (REALIST ORBCEL-C) was developed and patented by Orbsen Therapeutics.

Main outcomes: The primary safety outcome is the incidence of serious adverse events. The primary efficacy outcome is Oxygenation Index (OI) at day 7. Secondary outcomes include: Ol at days 4 and 14; respiratory compliance, driving pressure and $\mathrm{PaO}_{2} / \mathrm{FiO}_{2}$ ratio (PF ratio) at days 4, 7 and 14; Sequential Organ Failure Assessment (SOFA) score at days 4, 7 and 14; extubation and reintubation; ventilation free days at day 28; duration of mechanical ventilation; length of ICU and hospital stay; 28-day and 90-day mortality.

Randomisation: After obtaining informed consent, patients will be randomised via a centralised automated 24-hour telephone or web-based randomisation system (CHaRT, Centre for Healthcare Randomised Trials, University of Aberdeen). Randomisation will be stratified by recruitment centre and by vasopressor use and patients will be allocated to REALIST ORBCEL-C or placebo control in a 1:1 ratio.

Blinding (masking): The investigator, treating physician, other members of the site research team and participants will be blinded. The cell therapy facility and clinical trials pharmacist will be unblinded to facilitate intervention and placebo preparation. The unblinded individuals will keep the treatment information confidential. The infusion bag will be masked at the time of preparation and will be administered via a masked infusion set.

Numbers to be randomised (sample size): A sample size of 60 patients with 30 patients randomised to the intervention and 30 to the control group. If possible, recruitment will continue beyond 60 patients to provide more accurate and definitive trial results. The total number of patients recruited will depend on the pandemic and be guided by the data monitoring and ethics committee (DMEC).

Trial status: REALIST Phase 1 completed in January 2020 prior to the COVID-19 pandemic. This was an open label dose escalation study of REALIST ORBCEL-C in patients with ARDS. The COVID-19 pandemic emerged as REALIST Phase 2 was planned to commence and the investigator team decided to repurpose the Phase 2 trial as a COVID-19 specific trial. This decision was discussed and approved by the Trial Steering Committee (TSC) and DMEC. Submissions were made to the Research Ethics Committee (REC) and MHRA to amend the protocol to a COVID-19 specific patient population and the protocol amendment was accepted by the REC on $27^{\text {th }}$ March 2020 and MHRA on $30^{\text {th }}$ March 2020 respectively. Other protocol changes in this amendment included an increase in the time of onset of ARDS from 48 to 72 hours, inclusion of clinical outcomes as secondary outcomes, the provision of an option for telephone consent, an indicative sample size and provision to continue recruitment beyond this indicative sample size. The current protocol in use is version 4.0 23.03.2020 (Additional file 1). Urgent Public Health status was awarded by the NIHR on 2 April 2020 and the trial opened to recruitment and recruited the first participant the same day. At the time of publication the trial was open to recruitment at 5 sites across the UK (Belfast Health and Social Care Trust, King's College London, Guys and St Thomas' Hospital London, Birmingham Heartlands Hospital and the Queen Elizabeth Hospital Birmingham) and 12 patients have been recruited across these sites. Additional sites are planned to open and appropriate approvals for these are being obtained. It is estimated recruitment will continue for 6 months.

Trial registration: ClinicalTrials.gov NCT 03042143 (Registered 3 Feb 2017). EudraCT 2017-000585-33 (Registered 28 Nov 2017).

Full protocol: The full protocol (version 4.0 23.03.2020) is attached as an additional file, accessible from the Trials website (Additional file 1). In the interest of expediting dissemination of this material, the familiar formatting has been eliminated; this Letter serves as a summary of the key elements of the full protocol.

The study protocol has been reported in accordance with the Standard Protocol Items: Recommendations for Clinical Interventional Trials (SPIRIT) guidelines (Additional file 2).

Keywords: COVID-19, Randomised controlled trial, protocol, Mesenchymal Stromal Cells, Mesenchymal Stem Cells, MSCs, Acute Respiratory Distress Syndrome, ARDS 


\section{Supplementary information}

Supplementary information accompanies this paper at https://doi.org/10. 1186/s13063-020-04416-w.

Additional file 1. The REALIST Study Repair of Acute Respiratory Distress Syndrome by Stromal Cell Administration Protocol v 4.0.

Additional file 2. SPIRIT 2013 Checklist: Recommended items to address in a clinical trial protocol and related documents*.

\section{Acknowledgments}

The authors would like to acknowledge the support of the Wellcome Trust Health Innovation Challenge Fund [Reference 106939/Z/15/Z], the Research and Development Division of the Public Health Agency (Northern Ireland), members of the DMEC (Professor John Norrie, Professor Mervyn Singer and Professor Sam Janes) and members of the TSC (Professor Charles Hinds, Professor John Simpson, Professor Mike Grocott, Mr Barry Williams and Professor John Laffey).

\section{Authors' contributions}

DFM and CO'K conceived the study. All authors made a substantial contribution to the protocol development. All authors have read and approved the manuscript.

\section{Funding}

The Wellcome Trust Health Innovation Challenge Fund [Reference 106939/Z/15/Z] and the Research and Development Division of the Public Health Agency (Northern Ireland) will provide research costs for the REALIST study. The funders have no role in the study design, data acquisition, data analysis or manuscript preparation.

Orbsen have granted a non-exclusive, trial-specific licence to the Cellular and Molecular Therapies Division of the National Health Service Blood and Transplant Service to manufacture REALIST ORBCEL-C to GMP standards for the REALIST trial. Orbsen have no role in the study design, data acquisition, data analysis or manuscript preparation.

\section{Availability of data and materials}

The investigator team will have full access to the final trial dataset. The trial will be reported in accordance with the Consolidated Standards of Reporting Trials (CONSORT) guidelines. The results of the trial will be published when data on the primary outcome are available. Long term data and mechanistic data will also be reported though may form the basis of separate publications. Publications will be published in peer-reviewed open access journals (in keeping with the Wellcome Trust open access policy). A lay person's summary of the principal findings of the results will be sent to all patients involved in the study at their request.

\section{Ethics approval and consent to participate}

This trial has received ethics approval from the REC (North East York REC). Reference 18/NE/006. Favourable opinion received $6^{\text {th }}$ February 2018. REC approval for amended protocol (v4.0 23.03.2020) received $27^{\text {th }}$ March 2020. Informed consent will be obtained in accordance with the ethical principles which have their origin in the Declaration of Helsinki. Due to the incapacitating nature of the condition of participants in this trial, consent will be sought from either a Personal Legal Representative (PerLR) or Professional Legal Representative (ProLR). Given visiting restrictions in place during the COVID-19 pandemic provisions are in place to obtain verbal agreement from the PerLR via telephone. When participating patients regain capacity to understand the details of the trial, consent to continue participation in the study will be sought. Patients may withdraw or be withdrawn (by PerLR or ProLR) from the trial at any time without prejudice.

\section{Consent for publication}

Not applicable.

\section{Competing interests}

The authors declare that they have no competing interests.

\section{Author details}

${ }^{1}$ Wellcome Wolfson Institute for Experimental Medicine, School of Medicine, Dentistry and Biomedical Science Queen's University Belfast, Belfast, UK.
'Guy's and St Thomas' NHS Foundation Trust London and School of Immunology and Microbial Sciences, King's College London, London, UK. ${ }^{3}$ Research and Development lead in Critical Care, Kings Trauma Centre, King's College London, London, UK. ${ }^{4}$ Queen Elizabeth Hospital, Birmingham, UK. ${ }^{5}$ University of Warwick, Coventry, UK. ${ }^{6}$ Belfast Health and Social Care Trust, Belfast, UK. ${ }^{7}$ Royal Victoria Hospital, Belfast, UK. ${ }^{8}$ Northern Ireland Clinical Trials Unit, Belfast, UK. ${ }^{9} \mathrm{NHS}$ Blood and Transplant, Birmingham, UK. ${ }^{10}$ Independent Public and Patient Representative, Sherborne, UK. ${ }^{11}$ Royal College of Surgeons in Ireland, Dublin, Ireland. ${ }^{12}$ National University of Ireland, Galway, Ireland. ${ }^{13}$ School of Medicine, Dentistry and Biomedical Sciences, Queen's University Belfast, Belfast, UK.

Received: 12 May 2020 Accepted: 14 May 2020 Published online: 03 June 2020

\section{Publisher's Note}

Springer Nature remains neutral with regard to jurisdictional claims in published maps and institutional affiliations.

\section{Ready to submit your research? Choose BMC and benefit from:}

- fast, convenient online submission

- thorough peer review by experienced researchers in your field

- rapid publication on acceptance

- support for research data, including large and complex data types

- gold Open Access which fosters wider collaboration and increased citations

- maximum visibility for your research: over $100 \mathrm{M}$ website views per year

At $\mathrm{BMC}$, research is always in progress.

Learn more biomedcentral.com/submissions 